\title{
A Novel Monocyte Subset as a Unique Signature of Atherosclerotic Plaque Rupture
}

\author{
Ramona Vinci', Daniela Pedicino ${ }^{1,2 *}$, Alice Bonanni' ${ }^{1}$ Alessia D'Aiello ${ }^{1}$, Anna Severino', \\ Eugenia Pisano'2, Myriana Ponzo', Francesco Canonico', Pellegrino Ciampi' ${ }^{1}$, \\ Giulio Russo', Marianna Di Sario', Rocco Antonio Montone' ${ }^{2}$, Carlo Trani1,2, \\ Cristina Conte', Maria Chiara Grimaldi', Francesco Cribari' ${ }^{1}$, Massimo Massetti ${ }^{1,2}$, \\ Filippo Crea ${ }^{1,2}$ and Giovanna Liuzzo ${ }^{1,2}$ \\ 1 Department of Cardiovascular and Pneumological Sciences, Catholic University of the Sacred Heart, Rome, Italy, \\ ${ }^{2}$ Department of Cardiovascular Sciences, Fondazione Policlinico Universitario A. Gemelli IRCCS, Rome, Italy
}

OPEN ACCESS

Edited by:

Luca Liberale

University of Zurich, Switzerland

Reviewed by:

Simon Kraler,

University of Zurich, Switzerland

Aldo Bonaventura,

ASST Sette Laghi, Italy

Dario Bongiovanni,

Technical University of Munich,

Germany

*Correspondence:

Daniela Pedicino

danialla@gmail.com;

daniela.pedicino@policlinicogemelli.it

Specialty section

This article was submitted to

Signaling,

a section of the journal

Frontiers in Cell and Developmental

Biology

Received: 04 August 2021 Accepted: 03 September 2021

Published: 12 October 2021

Citation:

Vinci $R$, Pedicino $D$, Bonanni $A$, D'Aiello A, Severino A, Pisano E, Ponzo M, Canonico F, Ciampi P,

Russo G, Di Sario M, Montone RA,

Trani C, Conte C, Grimaldi MC

Cribari F, Massetti M, Crea F and Liuzzo G (2021) A Novel Monocyte

Subset as a Unique Signature

of Atherosclerotic Plaque Rupture.

Front. Cell Dev. Biol. 9:753223.

doi: 10.3389/fcell.2021.753223
The evaluation of monocyte subset distribution among acute coronary syndrome (ACS) patients according to culprit coronary plaque morphology has never been explored. We evaluated whether there were significant differences in frequency of circulating monocyte subsets isolated from ACS patients according to optical coherence tomography (OCT) investigation of plaque erosion and rupture. We enrolled 74 patients with non-STelevation ACS (NSTE-ACS), 21 of them underwent OCT investigation of the culprit coronary plaque and local macrophage infiltration (MØI) assessment. As control, we enrolled 30 chronic coronary syndrome (CCS) patients. We assessed the frequency of monocyte subsets in the whole study population, in reliance on their CD14 and CD16 expression (classical, CM: CD14 ${ }^{++} \mathrm{CD} 16^{-}$; intermediates, IM: CD14 ${ }^{++} \mathrm{CD} 16^{+}$; nonclassical, NCM: CD14 ${ }^{+} \mathrm{CD} 16^{++}$). Then, we tested the effect of lipopolysaccharide (LPS) (a CD14 ligand) on peripheral blood mononuclear cells (PBMCs) of NSTE-ACS patients, quantifying the inflammatory cytokine levels in cell-culture supernatants. Our data proved that monocyte subsets isolated from NSTE-ACS patients represent a peculiar biological signature of the pathophysiological mechanism lying beneath atherosclerotic plaque with a ruptured fibrous cap (RFC) as compared with plaque erosion. Moreover, the magnitude of LPS-mediated effects on IL-1 $\beta, I L-6$, and IL-10 cytokine release in cell-culture supernatants appeared to be greater in NSTE-ACS patients with RFC. Finally, we described a fourth monocyte population never explored before in this clinical setting (pre-classical monocytes, PCM: CD14 ${ }^{+} \mathrm{CD} 16^{-}$) that was prevalent in NSTE-ACS patients as compared with CCS and, especially, in patients with RFC and culprit plaque with MØl.

Keywords: monocyte subsets, acute coronary syndromes, plaque rupture, inflammation, innate immunity

\section{INTRODUCTION}

Innate immunity activation represents a key mechanism in the pathogenesis of acute coronary syndromes (ACS), involved in the onset and progression of the atherosclerotic plaques (Shalhoub et al., 2011). Among the innate immunity cells, monocytes are the most plastic and dynamic ones, being able to switch toward several functional phenotypes (Canè et al., 2019). About 
two-thirds of ACS patients presenting with plaque rupture at optical coherence tomography (OCT) investigation had evidence of plaque macrophage infiltration (MØI) and raised systemic levels of inflammatory biomarkers (Scalone et al., 2017). In ACS patients with ruptured fibrous cap (RFC), monocytederived macrophages display thrombotic and inflammatory activity (Fracassi et al., 2021). Moreover, besides the monocyteto-macrophage transition (Tabas and Lichtman, 2017), relying on CD14 (LPS co-receptor) and CD16 (Fc receptor FcRIII) surface expressions, three major monocyte subsets have been typically recognized, i.e., classical $\left(\mathrm{CM}: \mathrm{CD}_{14}{ }^{++} \mathrm{CD} 16^{-}\right)$, intermediate (IM: $\mathrm{CD} 14^{++} \mathrm{CD}^{+} 6^{+}$), and non-classical (NCM: $\mathrm{CD}_{14}{ }^{+} \mathrm{CD}_{16}{ }^{++}$) (Passlick et al., 1989; Ziegler-Heitbrock et al., 2010), each one exhibiting a different pathophysiological role (Tsujioka et al., 2009; Ghattas et al., 2013; Mossanen et al., 2020; Oh et al., 2021). Our purpose was to evaluate monocyte subset distribution in NSTE-ACS patients according to OCT identification of plaque rupture and erosion.

\section{MATERIALS AND METHODS}

\section{Population, Sample Collection, and Cell Isolation}

Our population included 30 chronic coronary syndrome (CCS) patients and 74 patients with non-ST-elevation acute coronary syndromes (NSTE-ACS) (Knuuti et al., 2020; Collet et al., 2021). Characteristics of the study population are presented in Table 1, while patient allocation in each experimental condition is schematized in Figure 1. All patients gave their written informed consent. The local Ethics Committee approved the study. Exclusion criteria were patient age over 80 years; heart failure with reduced ejection fraction (HFrEF) [left ventricular ejection fraction (EF) <40\%; New York Heart Association (NYHA) class III-IV] (Ponikowski et al., 2016); moderate to severe heart valve disease, both stenosis and regurgitation (inferred according to the ESC Guidelines, Baumgartner et al., 2017); in-stent restenosis cases or stent thrombosis; culprit saphenous vein graft lesions and more generally by-pass graft lesions (including left internal mammary artery, LIMA) due to the different pathogenic mechanisms with respect to native coronary lesions; anti-inflammatory or immunosuppressive therapies other than low-dose aspirin; autoimmune diseases; evidence of immunologic disorders or chronic infectious diseases; liver diseases (class A-C of CHILD-PUGH), malignancies; chronic kidney disease at stage 4 ; and recent major surgical procedures or trauma.

Eighteen milliliters of venous blood were obtained at $9 \pm 3 \mathrm{~h}$ from hospital admission and collected in ethylenediaminetetraacetic acid (EDTA) tubes. Peripheral blood mononuclear cells (PBMCs) were isolated at 2,200 $\times g$ and $25^{\circ} \mathrm{C}$ for $25 \mathrm{~min}$ through density gradient centrifugation methods (Lympholyte ${ }^{\circledR}$-H Cell Separation Media, CEDARLANE). PBMC pellets were washed and suspended in Dulbecco's phosphatebuffered saline (DPBS) (GIBCO; Invitrogen, Carlsbad, CA, United States) and used according to scheduled protocol steps
TABLE 1 | Baseline characteristics of the study population.

\begin{tabular}{|c|c|c|c|}
\hline & $\begin{array}{l}\text { Ccs } \\
n=30\end{array}$ & $\begin{array}{c}\text { NSTE-ACS } \\
n=74\end{array}$ & $P$ value \\
\hline Age, mean $\pm S D$ & $69 \pm 8.7$ & $65 \pm 12.9$ & 0.131 \\
\hline Gender, M/F & $22 / 8$ & $54 / 20$ & 0.970 \\
\hline \multicolumn{4}{|l|}{$\mathrm{CV}$ risk factors } \\
\hline Smoking (\%) & $19(63)$ & $46(62)$ & 0.911 \\
\hline Diabetes (\%) & $15(50)$ & $23(33)$ & 0.069 \\
\hline Hypertension (\%) & $26(87)$ & $55(74)$ & 0.168 \\
\hline Dyslipidemia (\%) & $16(53)$ & $38(51)$ & 0.855 \\
\hline Obesity (\%) & $7(23)$ & $12(16)$ & 0.395 \\
\hline Family history (\%) & $9(30)$ & $25(34)$ & 0.782 \\
\hline \multicolumn{4}{|l|}{ Previous history } \\
\hline ACS (\%) & $8(27)$ & $28(38)$ & 0.359 \\
\hline Previous PCl (\%) & $8(27)$ & $23(31)$ & 0.299 \\
\hline Previous CABG (\%) & $0(0)$ & $5(7)$ & 0.320 \\
\hline \multicolumn{4}{|l|}{ In-hospital management } \\
\hline $\mathrm{LVEF} \geq 50 \%(\%)$ & $23(77)$ & $55(74)$ & 0.999 \\
\hline Multivessel disease (\%) & $7(23)$ & $29(39)$ & 0.171 \\
\hline $\begin{array}{l}\mathrm{PCl} \text { for the index event (\%) } \\
\mathrm{CABG} \text { for the index event (\%) }\end{array}$ & $\begin{array}{c}20(77) \\
5(17)\end{array}$ & $\begin{array}{l}58(7) \\
8(11)\end{array}$ & $\begin{array}{l}0.220 \\
0.510\end{array}$ \\
\hline \multicolumn{4}{|l|}{ Medical therapy } \\
\hline DAPT (\%) $)^{\#}$ & $10(33)$ & $29(39)$ & 0.944 \\
\hline ASA (\%) & $23(77)$ & $44(60)$ & 0.027 \\
\hline Clopidogrel (\%) & $10(33)$ & $16(22)$ & 0.146 \\
\hline Prasugrel (\%) & 0 & $1(1)$ & 0.535 \\
\hline Ticagrelor (\%) & 0 & $15(20)$ & 0.009 \\
\hline Anticoagulants (\%) & 0 & $5(7)$ & 0.157 \\
\hline Beta-Blockers (\%) & $20(67)$ & $41(55)$ & 0.301 \\
\hline Diuretics (\%) & $5(17)$ & $13(17)$ & 0.981 \\
\hline ACE-I (\%) & $15(50)$ & $30(40)$ & 0.375 \\
\hline ARBs (\%) & $3(10)$ & $20(27)$ & 0.075 \\
\hline Statins (\%) & $21(70)$ & $42(57)$ & 0.086 \\
\hline Calcium-channel blockers (\%) & $6(20)$ & $8(11)$ & 0.166 \\
\hline Nitrates (\%) & 0 & $1(1)$ & 0.535 \\
\hline Insulin (\%) & $5(17)$ & $7(9)$ & 0.232 \\
\hline Oral antidiabetic (\%) & $8(27)$ & $15(20)$ & 0.356 \\
\hline \multicolumn{4}{|l|}{$\begin{array}{l}\text { Laboratory assay } \\
\text { (mean } \pm \text { SD) }\end{array}$} \\
\hline Total cholesterol (mg/dL) & $157 \pm 41$ & $158 \pm 40$ & 0.870 \\
\hline LDL (mg/dL) & $91 \pm 33$ & $97 \pm 34$ & 0.620 \\
\hline $\mathrm{HDL}$ (mg/dL) & $41 \pm 9$ & $41 \pm 14$ & 0.910 \\
\hline Triglycerides (mg/dL) & $125 \pm 44$ & $130 \pm 42$ & 0.690 \\
\hline Monocyte count $\left(10^{9} / \mathrm{L}\right)$ & $0.5 \pm 0.2$ & $0.6 \pm 0.2$ & 0.146 \\
\hline Monocyte count (\%) & $6.5 \pm 2.2$ & $6.3 \pm 1.7$ & 0.685 \\
\hline hs-CRP (mg/L) (median and IRQ) & $2.9 \pm 12.6$ & $10.5 \pm 23.3$ & 0.039 \\
\hline \multicolumn{4}{|l|}{ Follow-up events } \\
\hline $\begin{array}{l}\text { Recurrence of acute coronary } \\
\text { events (\%) }\end{array}$ & $6(20)$ & $33(45)$ & 0.019 \\
\hline Cardiovascular death & 0 & $2(3)$ & - \\
\hline Non-fatal Ml & $2(7)$ & $14(19)$ & - \\
\hline Ischemia-driven revascularization & $4(13)$ & $17(23)$ & - \\
\hline
\end{tabular}

\#These data refer to the time of patient enrollment and blood withdrawal. At the time of coronary angiography all the NSTE-ACS patients were on DAPT according to current guidelines.

Recurrence of acute coronary events means occurrence of cardiovascular death, non-fatal myocardial infarction, and ischemia-driven revascularization at 6-24 months of follow-up. Follow-up visits, consisting of physical examination, a standard 12-lead electrocardiogram, and a treadmill stress test were performed every 6 months.

CCS, chronic coronary syndromes; NSTE-ACS, non-ST-elevation acute coronary syndromes; $S D$, standard deviation; M/F, male/female; $C V$, cardiovascular; $P C l$, percutaneous coronary intervention; CABG, coronary artery bypass grafting; $L V E F$, left ventricular ejection fraction; DAPT, dual antiplatelet therapy; ASA, aspirin; ACEI, Angiotensin-converting enzyme inhibitors; ARBs, angiotensin II receptor blockers; $L D L$, low-density lipoprotein; HDL, high-density lipoprotein; hs-CRP, high sensitive $C$-reactive protein; IQR, interquartile range; MI, myocardial infarction. 


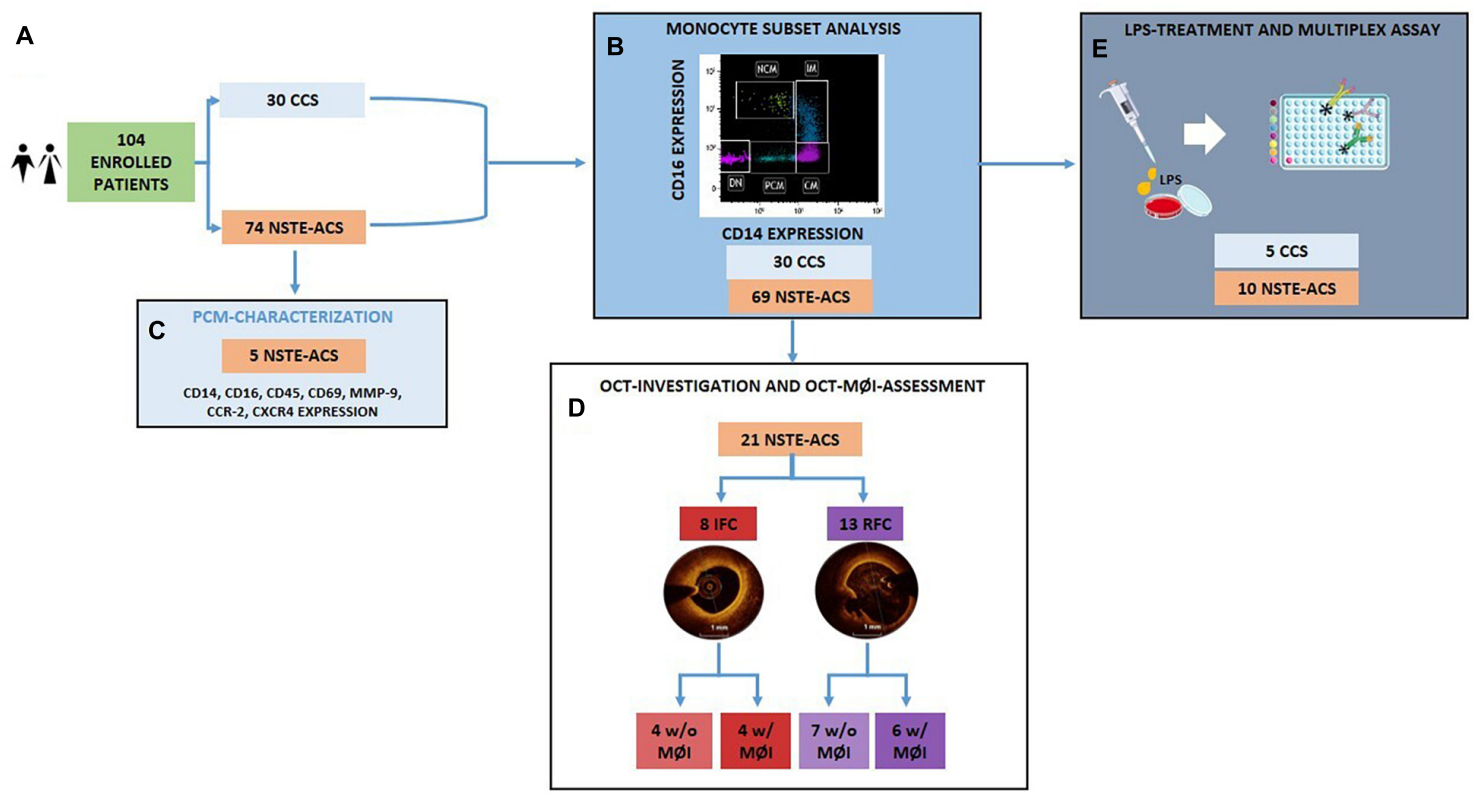

FIGURE 1 | Flowchart showing the allocation of the enrolled patient population within each experimental procedure. (A) Among the 104 enrolled patients, 30 were CCS and 74 were NSTE-ACS; (B) The analysis of distribution of circulating monocyte subsets was performed according to CD14 and CD16 expression on 30 CCS and 69 NSTE-ACS patients; (C) 5 new NSTE-ACS patients were enrolled to characterize a novel monocyte subpopulation; (D) 21 of the 69 NSTE-ACS patients underwent OCT investigation and OCT-MØI assessment, resulting in 8 IFC and 13 RFC, respectively; (E) Lastly, 5 CCS and 10 NSTE-ACS patients of the 30 CCS and 69 NSTE-ACS, respectively, were employed for the in vitro LPS-treatment and cytokine multiplex assay. CCS, chronic coronary syndrome, IFC, intact fibrous cap; LPS, lipopolysaccharides; MØI, machrophage infiltration; NSTE-ACS, non ST-elevation acute coronary syndrome; PBMCs, peripheral blood mononuclear cells; RFC, ruptured fibrous cap; w/o, without; w/with. Art images from http://smart.servier.com.

(see sections "Flow Cytometry Analysis" and "Cell Cultures and Multiplex Assay Analysis”).

\section{Optical Coherence Tomography Investigation and Optical Coherence Tomography-Macrophage Infiltration Assessment}

Optical Coherence Tomography images were obtained using a frequency domain OCT system (C7-XR FD-OCT; St. Jude Medical, St. Paul, MN, United States). OCT image analysis was performed using an offline review workstation (Ilumien Optis; St Jude Medical) by two expert investigators who were blinded to patient data. In presence of any diagnostic discordance between the two readers, a consensus was achieved taking into account the opinion of a third investigator. Plaque erosion has been classified by the presence of intra-luminal thrombus overlying a plaque presenting with intact fibrous cap (IFC), otherwise the presence of an irregular luminal surface at the site of the culprit lesion in the absence of thrombus. RFC has been defined by the existence of fibrous cap discontinuity with a cavity formation inside the plaque, or with a direct communication between the lumen and inner core of the lesion (Prati et al., 2010; Tearney et al., 2012; Johnson et al., 2019). Furthermore, the presence of macrophage infiltrations (MØI) at the culprit sites was assessed by OCT imaging. OCTMØI appeared as "signal-rich, distinct, or confluent punctate regions that exceed the intensity of background speckle noise and generate a backward shadowing" (Phipps et al., 2015; Montone et al., 2020).

Among our NSTE-ACS patients who underwent OCT investigation of culprit coronary lesions before stent procedure for clinical reasons and in whom it was possible to clearly identify the features of the culprit plaques, eight presenting with IFC and 13 with RFC have been identified. Patients were then divided into four groups according to the presence or absence of MØI as follows: four IFC without MØI, four IFC with MØI, seven RFC without MØI, and six RFC with MØI.

\section{Flow Cytometry Analysis}

The frequency of circulating monocyte subsets was assessed on basal PBMCs (30 CCS and 69 NSTE-ACS, of which 8 IFC and 13 RFC) by flow cytometry (Cytomics FC-500; Beckman Coulter, Brea, CA, United States). Monocyte staining with fluorochromelabeled monoclonal antibodies (mAbs) was performed for $15 \mathrm{~min}$ at room temperature (RT) and under dark condition using anti-CD14 electron coupled dye (ECD)-conjugated and anti-CD16 phycoerythrin cyanin 7 (PC7)-conjugated (both from Beckman Coulter). Monocytes were gated using a forward/sideward scatter (FS/SS) plot, and monocyte subsets were defined according to their cluster of differentiations (CD) CD14 and CD16 surface expression (Figure 1A). Furthermore, to distinguish the monocyte populations, additional cell surface expressions of CD14, CD16, CD45, CD69, matrix metalloproteinase 9 (MMP-9), C-C chemokine receptor 2 (CCR2), and C-X-C motif chemokine receptor 4 (CXCR4) 
were simultaneously evaluated in five patients presenting with cardiovascular diseases using a multi-color detector configuration set up (CytoFlex S; Beckman Coulter). The relative median fluorescence intensity (MFI) was assessed, and monocyte subset distribution was shown as frequency (\%) of each subset among the monocyte group according to gating strategy. Non-specific staining with isotype-matched control $\mathrm{mAb}$ was $<1 \%$. For each acquisition, 50,000 events were captured, and they were adequate for rare population analysis. Acquired data were analyzed using the Kaluza analysis software (Beckman Coulter).

\section{Cell Cultures and Multiplex Assay Analysis}

PBMCs were incubated at a density of $2 \times 10^{6} / \mathrm{ml}$ for $16 \mathrm{~h}$ at $37^{\circ} \mathrm{C}$ under $5 \% \mathrm{CO}_{2}$ and $20 \% \mathrm{O}_{2}$ in cell culture medium (RPMI 1640, LONZA) supplemented with $100 \mathrm{U} / \mathrm{ml}$ penicillin, $0.1 \mathrm{mg} / \mathrm{ml}$ streptomycin, $2 \mathrm{mmol}$ glutamine and $10 \%$ fetal bovine serum (FBS; Thermo Fisher Scientific, Carlsbad, CA, United States) and treated (or not) with $1 \mu \mathrm{g} / \mathrm{ml}$ Escherichia coli lipopolysaccharide (LPS, Sigma-Aldrich, S. Louis, MO, United States), as CD14 ligand as well as a known proinflammatory stimulus. We then analyzed the concentrations of six cytokines (interleukin-1 $\beta$, IL-1 $\beta$; interleukin-1r $\alpha$, IL1 r $\alpha$; interleukin-6, IL-6; interleukin-8, IL-8; interleukin-10, IL10 ; interferon- $\gamma$, IFN- $\gamma$ ) in collected supernatants from nontreated (NT) and LPS-treated PBMCs $(n=5$ CCS and $n=10$ NSTE-ACS, of which $n=4$ IFC and $n=6$ RFC) by using Bio-Plex ${ }^{\mathrm{TM}}$ Pro human 6-plex for group I cytokine (Bio-Rad Laboratories, Hercules, CA, United States) according to the protocol provided by the manufacturer. Data were collected and analyzed using a Bio-Rad BioPlex 200 instrument equipped with Bio-Plex Manager software version 6.0 (Bio-Rad Laboratories, Hercules, CA, United States). The precision based on both intra- and inter-assay variations were $<10 \%$ within the detection limits provided by the manufacturer.

\section{Statistical Analysis}

Due to the pioneering nature of the study, it was not possible to make a formal estimate of the power and sample size. Therefore, the enrollment of 30 patients in the CCS group and 74 patients in the NSTE-ACS group was arbitrary. However, based on very recent data (Merah-Mourah et al., 2020), the differences we observed should maintain statistical significance even with increasing sample size.

Variable distribution was assessed by Shapiro-Wilk test. For data normally distributed, we used unpaired $t$-test for statistics between two groups and one-way ANOVA for repeated measures followed by Holm-Sidak's multiple comparison test. Data that did not follow a normal distribution were analyzed using non-parametric tests, as follows: Mann-Whitney $U$ test was performed for statistics between two groups, and Wilcoxon and Friedman or Kruskal-Wallis test followed by Dunn's post hoc test for multiple comparisons. Mean \pm standard deviation (SD) and median and interquartile range (IQR) have been used for description of normally and non-normally distributed data, respectively. For all the experimental assays, a two-tailed $p \leq 0.05$ was considered statistically significant. The PRISM software (GraphPad 8.02; GraphPad Software Inc., San Diego, CA, United States) was used as statistics tool.

\section{RESULTS}

\section{Identification of a Novel Monocyte Subset}

Analysis of the distribution of circulating monocyte subsets according to their CD14 and CD16 surface expression led to the identification of a fourth monocyte population other than the three already known subsets (classical, CM: $\mathrm{CD} 14^{++} \mathrm{CD}^{-}{ }^{-}$; intermediate, IM: $\mathrm{CD}_{14}{ }^{++} \mathrm{CD}_{16}{ }^{+}$; non-classical, $\mathrm{NCM}$ : $\left.\mathrm{CD} 14^{+} \mathrm{CD} 16^{++}\right)$. In line with CD14 and CD16 surface staining, the newly identified cells were $\mathrm{CD}_{14}{ }^{+} \mathrm{CD}_{16}^{-}$(Figure 2
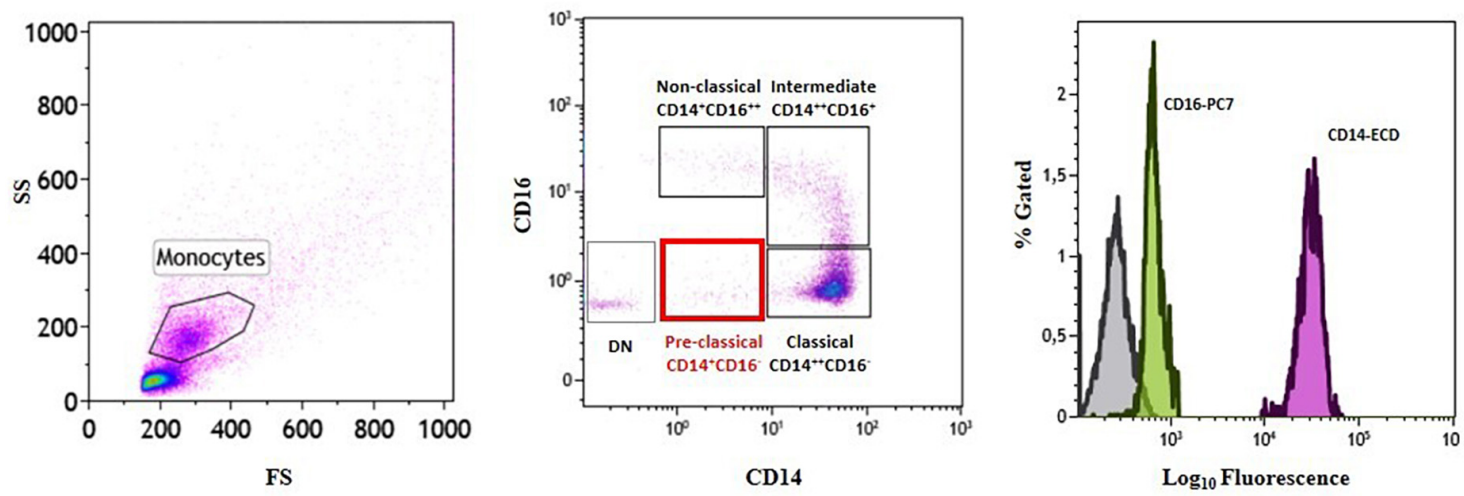

FIGURE 2 | Identification of a novel subset of circulating monocytes. Gating strategy and monocyte subset identification by flow cytometry in a representative patient Monocytes were gated in a forward/sideward scatter (FS/SS) dot plot (left plot) and the three monocyte subsets (classical, intermediate, and non-classical) (middle plot) as well as the novel pre-classical monocyte population were identified according to CD14 and CD16 cell surface expression (right histogram). DN = double negative. 
and Table 2). We decided to label this novel subset as "preclassical monocytes" (PCM) because of their proximity to the $\mathrm{CM}$ while observing the flow-cytometry acquired event distribution on dot plots.

Moreover, as shown in Supplementary Figure 1, to better distinguish the novel monocyte population of PCM from the known classification of CM, IM, and NCM, flow cytometry characterization of this subset was performed in patients presenting with NSTE-ACS $(n=5)$, in reliance on the expression of the following surface markers: CD14, CD16, CD45, CD69, MMP-9, CCR2, and CXCR4 (Table 2).

\section{Distribution of Circulating Monocyte Subsets in Non-ST-Elevation Acute Coronary Syndrome Patients and Its Relationship With Plaque Phenotype (Erosion Versus Rupture) and Local Macrophage Infiltration at Optical Coherence Tomography Investigation}

Interestingly, when monocyte subset distribution was evaluated in the whole study population, the newly identified PCM subset was more frequent in NSTE-ACS rather than in CCS patients (median, IQR: $5.2,6.2$ vs. $2.8,3.9 ; p=0.005$ ). NCM was more frequent in CCS rather than in NSTE-ACS patients (median, IQR: $5.2,3.6$ vs. $3.3,4.3 ; p=0.006$ ), while no significant differences were recorded comparing the CM (median, IQR: 67.0, 22.7 vs. 64.6, 19.6; $p=\mathrm{ns}$ ) and IM (median, IQR: $6.1,4.9$ vs. $6.5,4.8$; $p=\mathrm{ns}$ ) frequency distributions between CCS and NSTE-ACS patients ( $p>0.999$ for both). Nevertheless, CM was the most frequent monocyte subgroup in both CCS and NSTE-ACS, as expected according to the known monocyte subset distribution (Figure 3A).

When plaque phenotype was analyzed by OCT, NSTE-ACS patients with RFC plaques $(n=13)$ compared to patients with IFC plaque $(n=8)$ displayed a decreased frequency of CM (mean \pm SD: $60.1 \pm 13.4$ vs. $74.5 \pm 7.8, p=0.013$ ) and an increased frequency of NCM (median, IQR: 6.9, 7 vs. 2.0 2.0, $p=0.008)$. IM frequencies were similar between erosion and ruptured plaques $(p=0.886)$. The same analysis showed an increasing frequency of PCM in NSTE-ACS patients presenting

TABLE 2 | Monocyte phenotypic profiles.

\begin{tabular}{|c|c|}
\hline Monocyte subset & Phenotype \\
\hline $\begin{array}{l}\text { Pre-classical } \\
\text { monocyte }\end{array}$ & $\mathrm{CD}_{14}{ }^{+} \mathrm{CD} 16^{-} \mathrm{CD}_{4} 5^{+} \mathrm{CD} 69^{+} \mathrm{MMP}^{+} \mathrm{CCR}^{+}{ }^{+} \mathrm{CXCR}^{+}$ \\
\hline Classical monocyte & $\mathrm{CD}_{14}{ }^{++} \mathrm{CD} 16^{-} \mathrm{CD} 45^{+} \mathrm{CD} 9^{+} \mathrm{MMP9}^{+} \mathrm{CCR} 2^{+} \mathrm{CXCR}^{+}$ \\
\hline $\begin{array}{l}\text { Intermediate } \\
\text { monocyte }\end{array}$ & $\mathrm{CD} 14^{++} \mathrm{CD} 16^{+} \mathrm{CD} 45^{++} \mathrm{CD} 69+\mathrm{MMP}^{+}{ }^{\mathrm{CCR}} 2^{+} \mathrm{CXCR} 4^{+}$ \\
\hline $\begin{array}{l}\text { Non-classical } \\
\text { monocyte }\end{array}$ & $\mathrm{CD}_{14}{ }^{+} \mathrm{CD} 16^{++} \mathrm{CD} 45^{++} \mathrm{CD} 69^{+} \mathrm{MMP}^{+} \mathrm{CCR}^{+}{ }^{+} \mathrm{CXCR} 4^{+}$ \\
\hline \multicolumn{2}{|c|}{$\begin{array}{l}\text { A more accurate flow-cytometry characterization of monocyte subsets was } \\
\text { performed in five patients presenting with NSTE-ACS, according to the expression } \\
\text { of the following surface markers: CD14, CD16, CD45, CD69, MMP-9, CCR2, and } \\
\text { CXCR4. See also Supplementary Figure 1. }\end{array}$} \\
\hline
\end{tabular}

with RFC as compared with IFC lesions (median, IQR: 5.0, 10.1 vs. 3.2, 3.4; $p=0.054$ ) (Figure 3B). Representative dot plots of monocyte subset distribution in CCS and NSTE-ACS patients (Figure 3C) and in NSTE-ACS according to plaque phenotype (Figure 3D), and representative OCT images (Figure 3E) are shown.

Higher frequency of PCM was recorded in NSTE-ACS patients presenting plaque $\mathrm{M} \varnothing \mathrm{I}$ as compared to patients without local MØI (median, IQR: 7, 10.1 vs. 2.5, $3.3 p=0.013$ ), and specifically in NSTE-ACS patients with RFC lesions and MØI as compared to those with RFC lesions without MØI (median, IQR: 12.6, 15.1 vs. $2.5,3.3 ; p=0.011$ ), while no significant differences were recorded comparing the other three monocyte subsets (Figures $4 \mathrm{~A}-\mathrm{C}$ ).

\section{In Non-ST-Elevation Acute Coronary Syndrome Patients With Plaque Rupture, Lipopolysaccharide-Stimulation of Peripheral Blood Mononuclear Cells Triggers an Enhanced Cytokine Release}

The assessment of a cytokine panel by multiplex ELISA revealed that cultured PBMCs from NSTE-ACS patients respond to LPS treatment by releasing significant amounts of IL-1 $\beta$, IL-6, and IL-10 in the cell culture medium, as compared with non-treated cells (NT) $(p=0.008 ; p=0.016 ; p=0.008$, respectively), while no differences have been recorded for IL-1r $\alpha$, IL-8, and INF- $\gamma$ (Figure 5A). Although showing a release of IL-1 $\beta$, IL-6, and IL-10 in response to LPS stimulation, no significant differences has been recorded between NT and LPS-treated PBMCs from CCS patients for all the six cytokines studied. According to OCT analysis, the magnitude of LPS-mediated effects on IL1- $\beta$, IL-6, and IL-10 release in cell culture medium appeared to be greater in patients with RFC $(n=6)$, when compared to patients with IFC $(n=4)$, hence showing that these data have plaque specificity. Indeed, supernatants from PBMCs of RFC patients released the highest levels of IL-1 $\beta$ ( $p=0.035)$, IL-6 $(p=0.012)$, and IL-10 $(p=0.014)$ after LPS treatment, while no significant differences have been recorded within the IFC group (Figure 5B). No significant differences were found in high-sensitivity $\mathrm{C}$-reactive protein (hs-CRP) levels comparing IFC and RFC patient samples (see Supplementary Figure 2).

\section{DISCUSSION}

The pathophysiology of ACS is strictly related to an outburst of inflammatory (Biasucci et al., 2017) and immune-mediated response (Crea and Liuzzo, 2013), taking over the concept of the atheroma as a mere lipid-driven lesion (Soehnlein and Libby, 2021). Differences in innate and adaptive immune system have been described in ACS patients (Liuzzo et al., 2007, 2020; Flego et al., 2016; Leers et al., 2017; Pedicino et al., 2018a; Leistner et al., 2020). Starting from PBMCs as source material, our group documented an altered hyaluronan metabolism in NSTE-ACS patients presenting with plaque erosion (Pedicino et al., 2018b), further confirmed by later investigations on platelets and monocyte-platelet interaction (Vinci et al., 2021). 
A
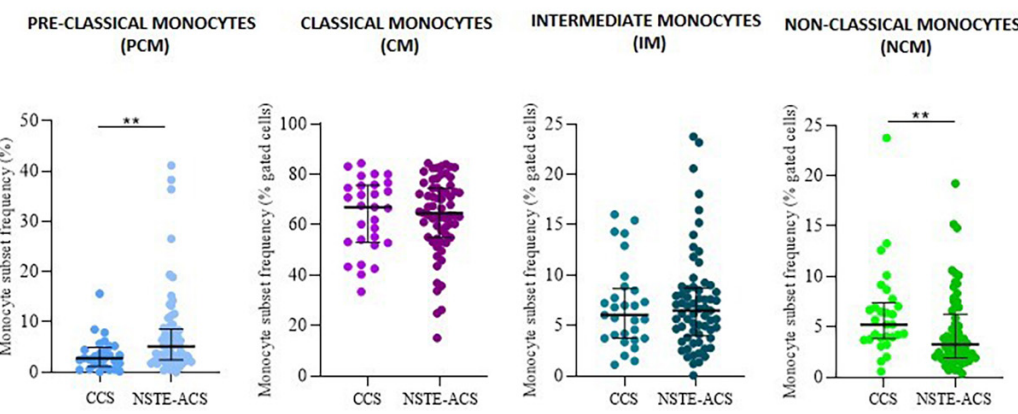

B
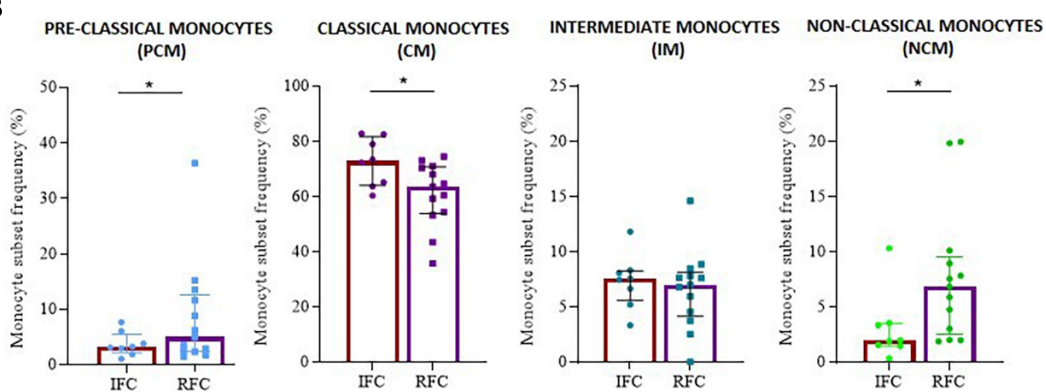

C

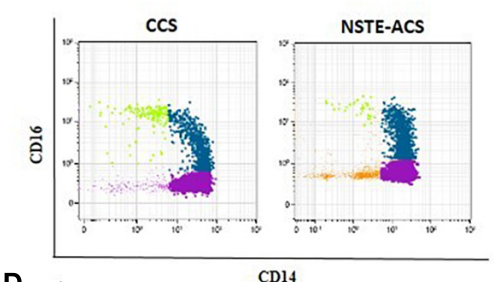

D

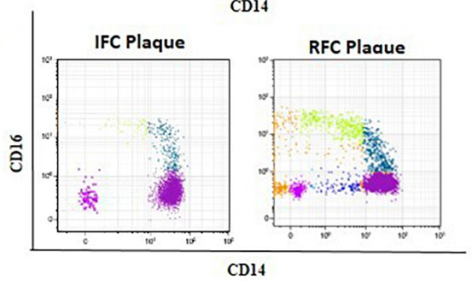

E

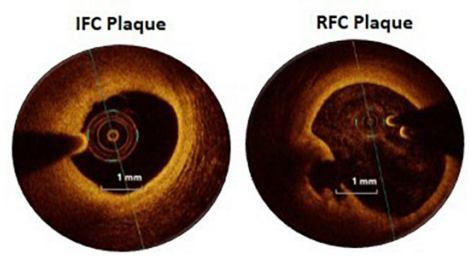

FIGURE 3 | Distribution of monocyte subsets between chronic coronary syndrome (CCS) and non-ST elevation acute coronary syndrome (NSTE-ACS) patients and its relationship with plaque phenotype at optical coherence tomography (OCT) investigation. (A) Frequency of pre-classical, classical, intermediate, and non-classical monocytes in CCS $(n=30)$ and NSTE-ACS $(n=69)$ patients and $(\mathbf{B})$ in NSTE-ACS patients with evidence of intact fibrous cap (IFC, $n=8)$ and ruptured fibrous cap (RFC, $n=13$ ) plaques according to OCT investigation. Two representative dot plots showing monocyte subset distribution (C) in CCS and NSTE-ACS patients and (D) in NSTE-ACS patients presenting with IFC and RFC plaques. (E) Two representative OCT images of IFC and RFC plaques. Data are presented as mean \pm SD; ${ }^{*} p<0.05,{ }^{* *} p<0.01$.

Those findings pointed the attention to the extracellular matrix derangement and its altered components (Angelini et al., 2018). On the other side, an expansion of $\mathrm{CD} 4{ }^{+} \mathrm{CD} 28^{\text {null }} \mathrm{T}$-cells represents distinctive features of NSTE-ACS patients with RFC lesions (Ruggio et al., 2019). These data suggest peculiar proinflammatory and immune-mediated mechanisms underneath different plaque morphologies.

Monocytes, as immune system early players, can receive alerts by infectious antigens through their surface pattern recognition receptors (PRRs) (Zanoni and Granucci, 2013) as well as by systemic and local pro-inflammatory signals (Shi and Pamer, 2011). Circulating monocyte subsets, whose detection is based on their surface expression of CD14 and CD16 antigens (Ziegler-Heitbrock et al., 2010), might have a variable distribution depending on the pathophysiological condition and their distinctive ability to plastic self-conversion (Gerrity, 1981; Stec et al., 2007; Kratofil et al., 2017; Roberts et al., 2020). Maladaptive innate immune plasticity, already recognized as a pivotal player in autoimmune diseases such as rheumatoid arthritis (Xue et al., 2020), systemic sclerosis, and systemic lupus erythematosus syndromes (Hirose et al., 2019; Ma et al., 2019), might fuel plaque fate and acute coronary events.

According to our multicolor flow-cytometry analyses, the decreased frequency of NCM in the peripheral blood of our NSTE-ACS patients as compared to CCS ones, beforehand proved by Flego et al. (2015), corroborated the downregulation of patrolling monocytes during an acute coronary event (Thomas et al., 2015; Leers et al., 2017). With this study, we aimed at investigating monocyte population distribution according to atherosclerotic plaque phenotyping.

Our data showed that NSTE-ACS patients presenting with RFC plaques exhibit a fall in the frequency of CM subset, alongside higher levels of circulating NCM. These findings might reflect a compensatory mechanism of $\mathrm{CM}$ consumption, on one side, and an increased demand of circulating NCM, on the other side, that, all in all, could serve as injury responders (Marsh et al., 2017). Our data are in line with the recent observation of the prevalence of pro-inflammatory monocytederived macrophages in ACS patients with plaque rupture (Fracassi et al., 2021).

Intriguingly, for the first time, we described a novel monocyte population in proximity of CM that we named preclassical monocyte. Our novel population resembles a recently identified monocyte subset defined as small $\mathrm{CD} 14^{+} \mathrm{CD} 16^{\text {neg }}$ through a comprehensive multicolor flow-cytometry study on healthy subjects (Merah-Mourah et al., 2020). The newly described PCM subset was higher in NSTE-ACS patients, in particular with RFC plaque and with concomitant presence of local macrophage infiltration (MØI) as assessed by OCT investigation. MØI represents one of the main initiators of atherosclerotic plaque onset and progression. Our group has previously demonstrated that not all ACS patients with plaque 

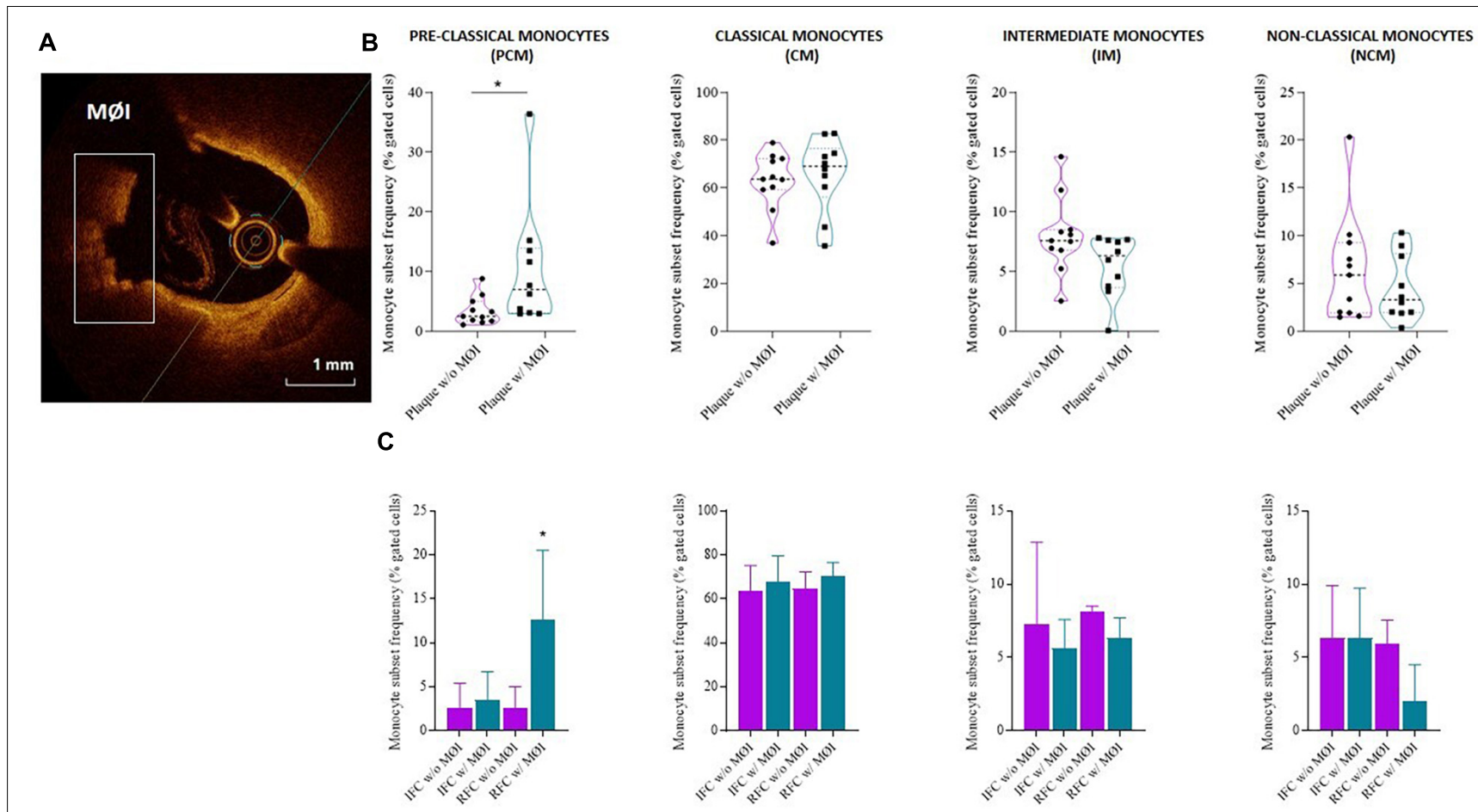

FIGURE 4 | Distribution of monocyte subsets in non-ST elevation acute coronary syndrome (NSTE-ACS) patients according to plaque erosion (IFC) or rupture (RFC) and local macrophage infiltration (MØI). (A) Representative optical coherence tomography (OCT) image of MØI. (B) Frequency of circulating monocyte subsets in NSTE-ACS patients according to OCT-MØI assessment, (C) and according to both plaque morphology (IFC versuS RFC) and MØI. Data are presented for panel (B) as median and interquartile range and for panel (C) as mean $\pm \mathrm{SD}$; ${ }^{*} p<0.05$.

rupture have local MØI, and that MØI is closely related with systemic inflammation (Scalone et al., 2017). Here we added another missing piece in the puzzle. Indeed, we found no differences in systemic hs-CRP levels between IFC and RFC, suggesting a similar systemic inflammatory burden in the two groups of patients. However, PCM expansion is strictly related to both MØI and RFC, while similar frequencies were observed in IFC and CCS, indicating an important role for this monocyte subset in the pathogenesis of plaque rupture at least in part independent from the systemic inflammatory burden (Biasucci et al., 2020). These data strongly suggest that alongside well recognized triggers of instability, such as systemic inflammation, other players are involved in the different fate of unstable plaque and that PCM might be used both as signature of plaque rupture and as promising targets for future therapeutic tools.

Finally, the LPS-treatment of circulating PBMCs of NSTE-ACS patients affects the pro-inflammatory (IL-1 $\beta$, IL-6) as well as anti-inflammatory (IL-10) cytokine release in cell-culture supernatants, especially in patients with RFC, suggesting that the intensity of the individual response to potential inflammatory stimuli may play a major role in determining the magnitude of the inflammatory reaction and clinical outcome (Liuzzo et al., 2001). These in vitro findings support our previous observation that NSTE-ACS with RFC are characterized by the expansion of an unusual subset of $\mathrm{T}$ cells committed to production of immunomodulatory cytokines, such as IL-12 and interferon (IFN)- $\gamma$ (Ruggio et al., 2019). As these cytokines induce a rapid priming of human monocyte functions, their chronic up-regulation could lead to subsequent activation of monocytes/macrophages in the circulation as well as in tissue lesions.

\section{STUDY LIMITATIONS}

Our study has some limitations. First, a formal estimate of the sample size was not pursued due to the innovative nature of the study. Since our work is a prospective analysis that includes a limited number of patients, we cannot exclude that the biological alterations described above may be part of the general stress response in the acute phase of ACS. Second, we based our plaque characterization on a small subset of enrolled NSTE-ACS patients who underwent OCT estimation for clinical reasons; study inclusion was also limited by the diagnostic certainty of OCT readers. Third, we tested the difference in inflammatory response following an exogenous proinflammatory stimulus, such as LPS, in patients with eroded and ruptured plaque, although we were aware of the small number of patients involved in this in vitro experiment due to sample availability and its allocation within each experimental phase. Finally, as the detection of macrophages by OCT raises technical limitations and consequent misdiagnoses, further validations with autopsy studies could be useful to confirm these results. 

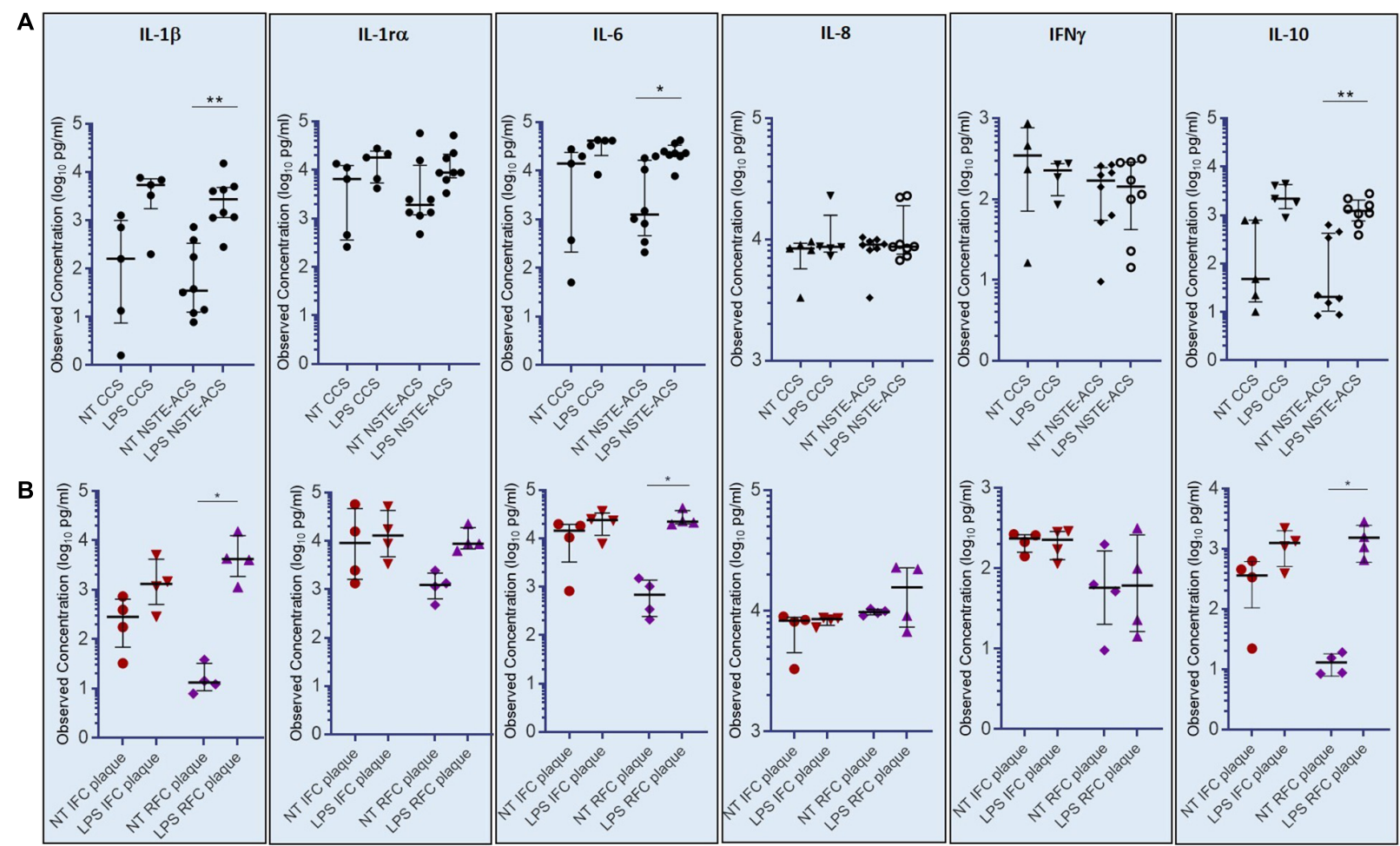

FIGURE 5 | Levels of cytokines (IL-1 $\beta$, IL-6, and IL-10) assessed in supernatants of cultured peripheral blood mononuclear cells (PBMCs) not-treated or treated with E. coli lipopolysaccharide (LPS). (A) Histograms showing observed cytokine concentrations ( $\mathrm{pg} / \mathrm{ml})$ in the supernatant of chronic coronary syndrome (CCS) and non-ST-elevation acute coronary syndrome (NSTE-ACS) PBMCs. (B) Histograms showing observed cytokine concentrations (pg/ml) in NSTE-ACS patients underwent to optical coherence tomography (OCT) investigation. Data are presented as median and interquartile range; ${ }^{*} p<0.05,{ }^{* *} p<0.01$.

We are aware that this is a descriptive and rather limited work, which needs to be confirmed in larger studies, and that the pathophysiology of our novel monocyte subset still needs to be properly investigated, conceivably using the singlecell technologies.

\section{CONCLUSION}

Circulating monocyte subsets of patients with NSTE-ACS show phenotypic heterogeneity with downregulation of patrolling monocytes and prevalence of inflammatory features in case of plaque rupture. Thus, monocyte subset diversity may take part in the cellular pathways leading to fibrous cap rupture and subsequent thrombus formation.

Of note, the present study is the first to investigate the pathophysiological role of a novel monocyte subpopulation that we named pre-classical monocyte (PCM) and to highlight a close relationship between PCM and plaque rupture with local macrophage infiltration. Alongside, the similar level of systemic inflammatory burden between patients presenting with RFC and IFC plaques, as indicated by CRP levels, confirms that mechanisms underlying plaque instability might be multiple and sometimes scarcely dependent on inflammatory bursts. In the era of precision medicine, different pathogenetic pathways might lead to ACS, and in this scenario, the higher frequency of PCM in patients with plaque rupture and local macrophage infiltration might represent a distinctive marker of a specific plaque phenotype, paving the way to novel diagnostic tools and tailored therapies for a selected group of patients. Indeed, in a translational perspective, the availability of a rapid blood test as a parallel biological, non-invasive screening that estimates the frequency of PCM during the patient hospital admission might be helpful for the consequent allocation of each patient toward even more personalized pharmacological therapies and/or interventional approaches (Crea and Liuzzo, 2016). Further specific studies are warranted in order to support our hypothesis.

\section{DATA AVAILABILITY STATEMENT}

The original contributions presented in the study are included in the article/Supplementary Material, further inquiries can be directed to the corresponding author/s.

\section{ETHICS STATEMENT}

The studies involving human participants were reviewed and approved by Ethics Committee of the Fondazione 
Policlinico Universitario “Agostino Gemelli” IRCCS- Università Cattolica del Sacro Cuore (UCSC), Rome (IT). The patients/participants provided their written informed consent to participate in this study.

\section{AUTHOR CONTRIBUTIONS}

RV and DP: conception and design. PC, MDS, CC, and FCr: provision of study materials and enrollment of patients. $\mathrm{RV}, \mathrm{AB}, \mathrm{EP}, \mathrm{AS}$, and FCa: collection of biological data and experimental activities. RV, DP, and GL: assembly of biological data. AD'A, MP, and MCG: collection and assembly of clinical data. CT, GR, and RM: hemodynamics investigation. RV, DP, and GL: data analysis and interpretation. GL, MM, and FiC: supervision. All authors approved the manuscript.

\section{REFERENCES}

Angelini, G., Flego, D., Vinci, R., Pedicino, D., Trotta, F., Ruggio, A., et al. (2018). Matrix metalloproteinase- 9 might affect adaptive immunity in non-ST segment elevation acute coronary syndromes by increasing CD31 cleavage on CD4 ${ }^{+}$ T-cells. Eur. Heart J. 39, 1089-1097. doi: 10.1093/eurheartj/ehx684

Baumgartner, H., Falk, V., Bax, J. J., De Bonis, M., Hamm, C., Holm, P. J., et al. (2017). 2017 ESC/EACTS guidelines for the management of valvular heart disease. Eur. Heart J. 38, 2739-2791. doi: 10.1093/eurheartj/ehx391

Biasucci, L. M., La Rosa, G., Pedicino, D., D’Aiello, A., Galli, M., and Liuzzo, G. (2017). Where does inflammation fit? Curr. Cardiol. Rep. 19:84. doi: 10.1007/ s11886-017-0896-0

Biasucci, L. M., Pedicino, D., and Liuzzo, G. (2020). Promises and challenges of targeting inflammation to treat cardiovascular disease: the post-CANTOS era. Eur. Heart J. 41, 2164-2167. doi: 10.1093/eurheartj/ehz586

Canè, S., Ugel, S., Trovato, R., Marigo, I., De Sanctis, F., Sartoris, S., et al. (2019). The endless saga of monocyte diversity. Front. Immunol. 10:1786. doi: 10.3389/ fimmu.2019.01786

Collet, J. P., Thiele, H., Barbato, E., Barthélémy, O., Bauersachs, J., Bhatt, D. L., et al. (2021). 2020 ESC Guidelines for the management of acute coronary syndromes in patients presenting without persistent ST-segment elevation. Eur. Heart J. 42, 1289-1367. doi: 10.1093/eurheartj/ehaa575

Crea, F., and Liuzzo, G. (2013). Pathogenesis of acute coronary syndromes. J. Am. Coll. Cardiol. 61, 1-11. doi: 10.1016/j.jacc.2012.07.064

Crea, F., and Liuzzo, G. (2016). Anti-inflammatory treatment of acute coronary syndromes: the need for precision medicine. Eur. Heart J. 30, 2414-2416. doi: 10.1093/eurheartj/ehw207

Flego, D., Liuzzo, G., Weyand, C. M., and Crea, F. (2016). Adaptive immunity dysregulation in acute coronary syndromes: from cellular and molecular basis to clinical implications. J. Am. Coll. Cardiol. 68, 2107-2117. doi: 10.1016/j.jacc. 2016.08.036

Flego, D., Severino, A., Trotta, F., Copponi, G., Manchi, M., Pedicino, D., et al. (2015). Reduced CD31 expression on CD14(+)CD16(+) monocyte subset in acute coronary syndromes. Int. J. Cardiol. 197, 101-104. doi: 10.1016/j.ijcard. 2015.06.039

Fracassi, F., Niccoli, G., Cosentino, N., Eligini, S., Fiorelli, S., Fabbiocchi, F., et al. (2021). Human monocyte-derived macrophages: pathogenetic role in plaque rupture associated to systemic inflammation. Int. J. Cardiol. 325, 1-8. doi: 10.1016/j.ijcard.2020.09.071

Gerrity, R. G. (1981). The role of the monocyte in atherogenesis: II. Migration of foam cells from atherosclerotic lesions. Am. J. Pathol. 103, 191-200.

Ghattas, A., Griffiths, H. R., Devitt, A., Lip, G. Y., and Shantsila, E. (2013). Monocytes in coronary artery disease and atherosclerosis: where are we now? J. Am. Coll. Cardiol. 62, 1541-1551. doi: 10.1016/j.jacc.2013. 07.043

\section{FUNDING}

The present study was partially supported by Catholic University of the Sacred Heart Linea D1 2018 Grant and by the Italian National Project Grant PRIN 2017, Protocol 2017WJBKKW_001.

\section{ACKNOWLEDGMENTS}

We thank participants for their important support for this study.

\section{SUPPLEMENTARY MATERIAL}

The Supplementary Material for this article can be found online at: https://www.frontiersin.org/articles/10.3389/fcell.2021. 753223/full\#supplementary-material

Hirose, S., Lin, Q., Ohtsuji, M., Nishimura, H., and Verbeek, J. S. (2019). Monocyte subsets involved in the development of systemic lupus erythematosus and rheumatoid arthritis. Int. Immunol. 31, 687-696. doi: 10.1093/intimm/dxz036

Johnson, T. W., Räber, L., Di Mario, C., Bourantas, C. V., Jia, H., Mattesini, A., et al. (2019). Clinical use of intracoronary imaging. Part 2: acute coronary syndromes, ambiguous coronary angiography findings, and guiding interventional decision-making: an expert consensus document of the European association of percutaneous cardiovascular interventions. EuroIntervention 15, 434-445. doi: 10.4244/EIJY19M06_02

Knuuti, J., Wijns, W., Saraste, A., Capodanno, D., Barbato, E., Funck-Brentano, C., et al. (2020). 2019 ESC guidelines for the diagnosis and management of chronic coronary syndromes. Eur. Heart J. 41, 407-477. doi: 10.1093/eurheartj/ehz425

Kratofil, R. M., Kubes, P., and Deniset, J. F. (2017). Monocyte conversion during inflammation and injury. Arterioscler. Thromb. Vasc. Biol. 37, 35-42. doi: 10.1161/ATVBAHA.116.308198

Leers, M. P. G., Stockem, C., Ackermans, D., Loeffen, R., Ten Cate, H., Kragten, J. A., et al. (2017). Intermediate and nonclassical monocytes show heterogeneity in patients with different types of acute coronary syndrome. Cytometry A 91, 1059-1067. doi: 10.1002/cyto.a.23263

Leistner, D. M., Kränkel, N., Meteva, D., Abdelwahed, Y. S., Seppelt, C., Stähli, B. E., et al. (2020). Differential immunological signature at the culprit site distinguishes acute coronary syndrome with intact from acute coronary syndrome with ruptured fibrous cap: results from the prospective translational OPTICO-ACS study. Eur. Heart J. 41, 3549-3560. doi: 10.1093/eurheartj/ ehaa703

Liuzzo, G., Angiolillo, D. J., Buffon, A., Rizzello, V., Colizzi, C., Ginnetti, F., et al. (2001). Enhanced response of blood monocytes to in vitro lipopolysaccharidechallenge in patients with recurrent unstable angina. Circulation 103, 22362241. doi: 10.1161/01.CIR.103.18.2236

Liuzzo, G., Biasucci, L. M., Trotta, G., Brugaletta, S., Pinnelli, M., Digianuario, G., et al. (2007). Unusual CD4+CD28null T lymphocytes and recurrence of acute coronary events. J. Am. Coll. Cardiol. 50, 1450-1458. doi: 10.1016/j.jacc.2007. 06.040

Liuzzo, G., Pedicino, D., Vinci, R., and Crea, F. (2020). CD8 lymphocytes and plaque erosion: a new piece in the jigsaw. Eur. Heart J. 41, 3561-3563. doi: 10.1093/eurheartj/ehaa721

Ma, W. T., Gao, F., Gu, K., and Chen, D. K. (2019). The role of monocytes and macrophages in autoimmune diseases: a comprehensive review. Front. Immunol. 10:1140. doi: 10.3389/fimmu.2019.01140

Marsh, S. A., Arthur, H. M., and Spyridopoulos, I. (2017). The secret life of nonclassical monocytes. Cytometry A 91, 1055-1058. doi: 10.1002/cyto.a.23280

Merah-Mourah, F., Cohen, S. O., Charron, D., Mooney, N., and Haziot, A. (2020). Identification of novel human monocyte subsets and evidence for phenotypic groups defined by interindividual variations of expression of adhesion molecules. Sci. Rep. 10:4397. doi: 10.1038/s41598-020-61022-1 
Montone, R. A., Vetrugno, V., Camilli, M., Russo, M., Fracassi, F., Khan, S. Q., et al. (2020). Macrophage infiltrates in coronary plaque erosion and cardiovascular outcome in patients with acute coronary syndrome. Atherosclerosis 311, 158166. doi: 10.1016/j.atherosclerosis.2020.08.009

Mossanen, J. C., Jansen, T. U., Pracht, J., Liepelt, A., Buendgens, L., Stoppe, C., et al. (2020). Elevated circulating CD14++CD16+ intermediate monocytes are independently associated with extracardiac complications after cardiac surgery. Sci. Rep. 10:947. doi: 10.1038/s41598-020-57700-9

Oh, E. S., Na, M., and Rogers, C. J. (2021). The association between monocyte subsets and cardiometabolic disorders/cardiovascular disease: a systematic review and meta-analysis. Front. Cardiovasc. Med. 8:640124. doi: 10.3389/fcvm. 2021.640124

Passlick, B., Flieger, D., and Ziegler-Heitbrock, H. W. (1989). Identification and characterization of a novel monocyte subpopulation in human peripheral blood. Blood 74, 2527-2534. doi: 10.1182/blood.V74.7.2527.2527

Pedicino, D., Giglio, A. F., Ruggio, A., Massaro, G., D’Aiello, A., Trotta, F., et al. (2018a). Inflammasome, T lymphocytes and innate-adaptive immunity crosstalk: role in cardiovascular disease and therapeutic perspectives. Thromb. Haemost. 118, 1352-1369. doi: 10.1055/s-0038- 1666860

Pedicino, D., Vinci, R., Giglio, A. F., Pisano, E., Porto, I., Vergallo, R., et al. (2018b). Alterations of hyaluronan metabolism in acute coronary syndrome: implications for plaque erosion. J. Am. Coll. Cardiol. 72, 1490-1503. doi: 10.1016/j.jacc.2018.06.072

Phipps, J. E., Vela, D., Hoyt, T., Halaney, D. L., Mancuso, J. J., Buja, L. M., et al. (2015). Macrophages and intravascular OCT bright spots: a quantitative study. JACC Cardiovasc. Imaging 8, 63-72. doi: 10.1016/j.jcmg.2014.07.027

Ponikowski, P., Voors, A. A., Anker, S. D., Bueno, H., Cleland, J. G. F., Coats, A. J. S., et al. (2016). 2016 ESC guidelines for the diagnosis and treatment of acute and chronic heart failure: the task force for the diagnosis and treatment of acute and chronic heart failure of the European society of cardiology (ESC) developed with the special contribution of the Heart failure association (HFA) of the ESC. Eur. Heart J. 37, 2129-2200. doi: 10.1093/eurheartj/ehw128

Prati, F., Regar, E., Mintz, G. S., Arbustini, E., Di Mario, C., Jang, I. K., et al. (2010). Expert review document on methodology, terminology, and clinical applications of optical coherence tomography: physical principles, methodology of image acquisition, and clinical application for assessment of coronary arteries and atherosclerosis. Eur. Heart J. 31, 401-415. doi: 10.1093/ eurheartj/ehp433

Roberts, M. E., Barvalia, M., Silva, J. A. F. D., Cederberg, R. A., Chu, W., Wong, A., et al. (2020). Deep phenotyping by mass cytometry and single-cell RNA-sequencing reveals LYN-regulated signaling profiles underlying monocyte subset heterogeneity and lifespan. Circ. Res. 126, e61-e79. doi: 10.1161/ CIRCRESAHA.119.315708

Ruggio, A., Pedicino, D., Flego, D., Vergallo, R., Severino, A., Lucci, C., et al. (2019). Correlation between CD4+CD28null T lymphocytes, regulatory $\mathrm{T}$ cells and plaque rupture: an optical coherence tomography study in acute coronary syndromes. Int. J. Cardiol. 276, 289-292. doi: 10.1016/j.ijcard.2018.08.101

Scalone, G., Niccoli, G., Refaat, H., Vergallo, R., Porto, I., Leone, A. M., et al. (2017). Not all plaque ruptures are born equal: an optical coherence tomography study. Eur. Heart J. Cardiovasc. Imaging 18, 1271-1277. doi: 10.1093/ehjci/jew208

Shalhoub, J., Falck-Hansen, M. A., Davies, A. H., and Monaco, C. (2011). Innate immunity and monocyte-macrophage activation in atherosclerosis. J. Inflamm. 8:9. doi: 10.1186/1476-9255-8-9

Shi, C., and Pamer, E. G. (2011). Monocyte recruitment during infection and inflammation. Nat. Rev. Immunol. 11, 762-774. doi: 10.1038/nri3070

Soehnlein, O., and Libby, P. (2021). Targeting inflammation in atherosclerosis from experimental insights to the clinic. Nat. Rev. Drug Discov. 20, 589-610. doi: 10.1038/s41573-021-00198-1
Stec, M., Weglarczyk, K., Baran, J., Zuba, E., Mytar, B., Pryjma, J., et al. (2007). Expansion and differentiation of CD14+CD16(-) and CD14+ +CD16+ human monocyte subsets from cord blood CD34+ hematopoietic progenitors. J. Leukoc. Biol. 82, 594-602. doi: 10.1189/jlb.0207117

Tabas, I., and Lichtman, A. H. (2017). Monocyte-macrophages and T cells in atherosclerosis. Immunity 47, 621-634. doi: 10.1016/j.immuni.2017. 09.008

Tearney, G. J., Regar, E., Akasaka, T., Adriaenssens, T., Barlis, P., Bezerra, H. G., et al. (2012). Consensus standards for acquisition, measurement, and reporting of intravascular optical coherence tomography studies: a report from the International working group for intravascular optical coherence tomography standardization and validation [published correction appears in J Am Coll Cardiol. 2012;59:1662. Dudeck, Darius [corrected to Dudek, Darius]; Falk, Erlin [corrected to Falk, Erling]; Garcia, Hector [corrected to Garcia-Garcia, Hector M]; Sonada, Shinjo [corrected to Sonoda, Shinjo]; Troels, Thim [corrected to Thim, Troels]; van Es, Gerrit-Ann [correct]. J. Am. Coll. Cardiol. 59, 10581072.

Thomas, G., Tacke, R., Hedrick, C. C., and Hanna, R. N. (2015). Nonclassical patrolling monocyte function in the vasculature. Arterioscler. Thromb. Vasc. Biol. 35, 1306-1316. doi: 10.1161/ATVBAHA.114.304650

Tsujioka, H., Imanishi, T., Ikejima, H., Kuroi, A., Takarada, S., Tanimoto, T., et al. (2009). Impact of heterogeneity of human peripheral blood monocyte subsets on myocardial salvage in patients with primary acute myocardial infarction. J. Am. Coll. Cardiol. 54, 130-138. doi: 10.1016/j.jacc.2009.04.021

Vinci, R., Pedicino, D., D’Aiello, A., Ciampi, P., Ponzo, M., Bonanni, A., et al. (2021). Platelet hyaluronidase 2 enrichment in acute coronary syndromes: a conceivable role in monocyte-platelet aggregate formation. J. Enzyme Inhib. Med. Chem. 36, 785-789. doi: 10.1080/14756366.2021.190 0159

Xue, J., Xu, L., Zhu, H., Bai, M., Xin, L., Zhao, Z., et al. (2020). CD14+CD16monocytes are the main precursors of osteoclasts in rheumatoid arthritis via expressing Tyro3TK. Arthritis Res. Ther. 22:221. doi: 10.1186/s13075-02002308-7

Zanoni, I., and Granucci, F. (2013). Role of CD14 in host protection against infections and in metabolism regulation. Front. Cell. Infect. Microbiol. 3:32. doi: $10.3389 /$ fcimb.2013.00032

Ziegler-Heitbrock, L., Ancuta, P., Crowe, S., Dalod, M., Grau, V., Hart, D. N., et al. (2010). Nomenclature of monocytes and dendritic cells in blood. Blood 116, e74-e80. doi: 10.1182/blood-2010-02-258558

Conflict of Interest: The authors declare that the research was conducted in the absence of any commercial or financial relationships that could be construed as a potential conflict of interest.

Publisher's Note: All claims expressed in this article are solely those of the authors and do not necessarily represent those of their affiliated organizations, or those of the publisher, the editors and the reviewers. Any product that may be evaluated in this article, or claim that may be made by its manufacturer, is not guaranteed or endorsed by the publisher.

Copyright (c) 2021 Vinci, Pedicino, Bonanni, D’Aiello, Severino, Pisano, Ponzo, Canonico, Ciampi, Russo, Di Sario, Montone, Trani, Conte, Grimaldi, Cribari, Massetti, Crea and Liuzzo. This is an open-access article distributed under the terms of the Creative Commons Attribution License (CC BY). The use, distribution or reproduction in other forums is permitted, provided the original author(s) and the copyright owner(s) are credited and that the original publication in this journal is cited, in accordance with accepted academic practice. No use, distribution or reproduction is permitted which does not comply with these terms. 